\title{
Manifestaciones afectivas en jóvenes con discapacidad intelectual*
}

\section{Affective manifestations in young people with intellectual disabilities}

\author{
Claudia Huaiquián Billeke**, Carolina Arriagada Vega***, Ada Betanzo Brio- \\ nes****, Hernán Inostroza Soto***** y Karina Llanquitruf Paillán******
}

\begin{abstract}
*Trabajo realizado en el marco del Proyecto PMI 1312 de la Facultad de Educación de la Universidad Católica de Temuco. Se incluyeron algunas conclusiones de la Tesis de Licenciatura en Educación denominada Manifestaciones afectivas en jóvenes con discapacidad, realizada por los cuatro coautores. **Doctora en Educación, Magister en Psicología y Psicopedagoga. Profesora de la Universidad Católica de Temuco. E-Mail: chuaiquian@uct.cl

***Licenciada en Educación y Profesora de Educación Diferencial. E-Mail: carolina.arriagada1993@gmail.com

****Licenciada en Educación y Profesora de Educación Diferencial. Profesora en la Escuela Manuel

Recabarren.Temuco - Chile. E-Mail: ada.betanzo@gmail.com

*****Licenciado en Educación y Profesor de Educación Diferencial. Profesor de Educación Diferencial en el Liceo Jorge Alessandri Rodriguez, Victoria, Chile. E-Mail: hernan4.inostroza@gmail.com ******Licenciada en Educación. Profesora de Educación Diferencial en el Colegio Leovigildo Kley. Cunco - Chile. E-Mail: karina.1lanquitruf@gmail.com
\end{abstract}

Universidad Católica de Temuco (UCT). Temuco - Chile.

\section{Resumen}

La afectividad en personas con discapacidad intelectual es un tema que ha sido omitido y ha generado prejuicios desde la ética cultural. La investigación que se informa consistió en develar las manifestaciones afectivas en jóvenes con discapacidad intelectual, con un rango de edad de 15 a 25 años. El nivel de conocimiento que alcanza la investigación, se enmarca en un estudio de tipo descriptivo denso.

La recolección de datos se realizó en una escuela especial de la Araucanía (Chile), aplicando entrevistas semiestructuradas con diseño estudio de caso. Se develó que las manifestaciones de afectividad de los jóvenes se sustentan en el cuidado y respeto mutuo, evidenciando creencias marcadas por la presencia de machismo, conciencia de responsabilidades y posicionamientos valóricos de adultos significativos que limitan, regulan comportamientos y omiten la educación sexual por la condición de los jóvenes, eviden- ciándose esta última de forma transversal al currículo de escuela especial.

Palabras clave: Discapacidad intelectual; Juventud; Afectividad; Sexualidad; Chile.

\begin{abstract}
This research studies the affectivity in people with intellectual disability condition, in order to visualize the process of self-construction of the sense of being person. This research looks for revealing the affective manifestations in young people with intellectual disability, whose ages are between 15 and 25 years old. This problem implies a complex issue, which involves cultural, religious, educative and idiosyncratic factors that reflect an unfair society which does not protect the rights of social groups in disadvantage situations and difficult educability conditions.

Theoretically is assumed an axiological positioning which conceives the person as a value in
\end{abstract}


itself and a spiritual microcosm which is alive and transcendent. The method applied is guided from the qualitative approach with a descriptive reach through the design associated to the case study and in accordance with the hermeneutic paradigm. The context of the problem was focus on a special school in the Araucanía region at the South of Chile. Semi-structure interviews and appropriate rigor criteria such as confirm ability, transferability, credibility and dependency were applied. The participants were 13 students and four teachers. The research is adjusted to the ethical requirements in the Helsinki and Singapore Declaration, according to the appropriate behavior of the researcher and the informed consent.

The qualitative data were reduced through the transcription and coding process using the Atlas.ti software version 7.0. With the primary transcribed documents axial and open coding were applied, which let to develop analysis from the constant comparative method. An empirical- theoretical sample and content analysis were done. The results let the categories raise, operationalized in codes, highlighting between them, affective manifestations, couple relationship, personal care, young people beliefs and teachers' beliefs. It was revealed that the affective manifestations of young people are based in the mutual respect and care, demonstrating beliefs with sexist characteristics, conscience of responsibilities and the meaningful adults' positions related to values, which limit and regulate behaviors and omit the sexual education because the condition that the young people have, evincing the sexual education in a transversal way to the curriculum of the special school.

The sexual education must give precise information and not be restricted to the intellectual agreement, but respecting the appropriate ages of the people life cycle, where the education must be precise and direct. To accomplish this task, it was discovered that didactic resources must be given to help young people to be aware of their action. Additionally, it was revealed that young people must be constantly accompanied by guides, mediation regarding to the construction of bonds and affective experience suitable to their own characteristics. Family is presented as an obstructive element in the young people affective relationships, evincing a lack of communication and comprehension. Teachers consider the young people as valuable people, where their intellectual conditions do not affect their rights to manifest themselves affectively either their friends or partners.

In summary, it must advance toward an education that overcomes the existence of false beliefs, and that will exist in the sexual education the disposition to talk with respect about what the young people say their interests, worries, responsibilities and respect with themselves and with others. For this reason, it is necessary to guide and orient young people, not only from the formal educational institution, but also from the collaborative work with the family, where this formation should not be delegated only to the professionals, but also that it constitutes in a contextualized learning of sexual education and that it must be accepted as an inherent condition from the human being, so that, in this way it must be experienced the liberty of the rights and the ethical personalism of each human being.

Key words: Intellectual disability; Youth, Affectivity, Sexuality.

\section{Introducción}

Las manifestaciones de afectividad de los jóvenes permiten el desarrollo digno, libre y responsable de las personas; reconocerlos ayuda a eliminar prejuicios que actualmente existen con respecto a esta temática. La persona con discapacidad intelectual es un ser humano que ama, se comunica, se relaciona, siente, experimenta placer y toma decisiones como cualquier individuo, ya que la presencia de discapacidad intelectual no implica alteración del desarrollo sexual y afectivo.

En este orden de cosas, los procesos afectivos proveen valores orgánicos naturales como conductores del comportamiento. Las manifestaciones afectivas se evidencian como un conocimiento directo de realidad interna, afectada por exigencias externas, permitiendo la autorregulación e identificando siete sistemas afectivos, tales como: búsqueda, ira, temor, deseo sexual, cuidado del otro, pánico, aflicción y juego (Panksepp, 2011). 
Es evidente que resolver estas cuestiones tiene que ver con la habilidad de los investigadores, pero igualmente es necesario asumir los desafíos que estas miradas implican. No se trata solamente de resolver estas preguntas sobre el acercamiento metodológico (que a la vez tienen implicaciones éticas y morales), sino también desde los aspectos emocionales y las vivencias cotidianas de cada uno de sus miembros. A la vez se fortalece una mirada social de la discapacidad, la cual no se logra fácilmente con otras formas de investigación, en la medida que captan la riqueza y los detalles de los significados en los asuntos humanos, motivaciones, sentimientos, deseos entre otros (Pava-Ripoll, 2015).

Aguirre et al. (2011), Pallisera et al. (2013) y Reisin (2010) respaldan el fortalecimiento de la calidad de vida, evitando infantilizar a las personas con discapacidad intelectual en las relaciones que establecen con los otros, entregando apoyos y orientaciones para la autonomía y participación social.

Kijak (2011), Olavarrieta y colaboradores (2013), Katz y Lazcano (2008), Ferreira (2014) y Abreu (2010) plantean que los hombres y mujeres con discapacidad intelectual son seres sexuados, que pueden procrear, por tanto, portadores conscientes de su propia intimidad. Esta área es un asunto complejo, ya que implica factores culturales, religiosos, educativos e idiosincrásicos, que reflejan una sociedad injusta al no proteger los derechos de grupos sociales en situación de desventaja.

León (2012) y Panksepp (2011) plantean que los procesos afectivos regulan los perceptivos, tienen una aparición rápida e independiente de la evaluación cognitiva y son las primeras reacciones del organismo, generando acciones dominantes. A la vez, presen$\tan$ valores subjetivos que apoyan elecciones conductuales. León, Bolaños, Campos y Mejías (2013) y Sim-Sim (2013) afirman que la pasión es una expresión de afectividad, parte del desarrollo humano y en los jóvenes se pueden vislumbrar situaciones de riesgo como prácticas de sexo inseguro, al no tener claridad en los límites para demostrar su afectividad. Estudios demuestran la no existencia de correlaciones significativas en la totali- dad de las tareas involucradas para reconocimiento de emociones básicas y complejas; no es posible concluir que las emociones básicas están relacionadas con las secundarias. Se infiere entonces que las emociones básicas son un tipo cualitativamente distinto de estado emocional que las emociones secundarias (Tabernero \& Politis, 2016).

Estudios revisados por Mateos y colaboradores (2014) y Vianchá, Bahamón, Tobos, Alarcón, Uribe y Alvarado (2012) plantean un enfoque ecológico de sexualidad señalando aspectos afectivos, sociales y culturales que condicionan, los centros de educación son el lugar en que los jóvenes reciben formación afectiva y sexual. En este sentido, los profesores deben conocer las características y necesidades individuales, para otorgar adecuadas orientaciones. Janeiro, Oliveira, Rodrigues, Maceiras y Rocha (2013) y Vargas-Barrantes (2013) concluyen que los hombres poseen actitudes más permisivas, mayor comunicación sexual, liberación y están más a favor del placer físico que las mujeres.

En Latinoamérica, Uribe, Amador, Zacarías y Villarreal (2012), Coutts y Morales (2011), Chávez y Álvarez (2012) y Malón (2012) plantean que la sexualidad más que un deber saber o deber conocer, se asume como un discurso social, donde se reconocen como individuos activos en la realidad contextual.

Por tanto, la educación sexual es una formación pendiente, entrecruzada de desacuerdos, debates y escepticismos que complican su aplicación en el sistema escolar; espacio que se constituye en un dispositivo para regular el comportamiento sexual.

Vargas Barrantes (2013), Viera Cherro (2011) y Vived Conte, Mullet, Díaz Orgaz, González-Simancas y Matía Amor (2013) sintetizan que los profesores de educación especial preparan a los estudiantes para una vida adulta con programas sistemáticos, evitando la afectividad, entregando conocimientos superficiales y limitantes sin argumentos científicos y enfatizando en la didáctica como ruptura epistemológica con respecto a la educación sexual tradicional.

Para Mora Huerta y Rodríguez Calvillo (2011), González, Molina, Montero y Martí- 
nez (2011), Rodríguez Cabrera, Sanabria Ramos, Contreras Palú y Pardomo Cáceres (2013), Caricote (2010) y Navarro y Hernández (2012) la educación sexual óptima se debe abordar a partir del manejo de emociones, dignidad, autoconocimiento, intimidad personal para dirigir de forma más sana y segura sus comportamientos y con estrategias centradas en las necesidades de adolescentes, factores personales y familiares. Asimismo, plantean que las dificultades en el conocimiento del comportamiento de salud sexual y reproductiva derivan en conductas de riesgos.

Belmonte y García (2014) afirman que los adultos tienen menos apertura hacia la sexualidad de personas con discapacidad intelectual, por tanto, el factor de edad interviene en las actitudes hacia el tema y aceptación. Complementando estudios de Caricote (2010, 2012), Aya Gómez y Córdoba (2013), Silveira Araujo y Rossito Aiello (2013) y Cosentino, Maia, Rodrigues, Rossetto y Silva (2010) señalan que las oportunidades para ampliar el conocimiento con respecto a la sexualidad en personas con discapacidad intelectual se encuentran limitadas por programas de promoción sexual sana y segura en instituciones educativas, sin considerar los roles sociales y recayendo en la entrega de información repetida asociada a la prevención.

Campbell y Gilmore (2014), Mattos Caldeira, Rajab, Cardia, Bicalho Reis y da Silva Ferrao (2012) y Mella (2012) concluyen que la sexualidad en personas con discapacidad intelectual presenta prejuicios, mistificación y discriminación, incluso en contextos familiares. Siendo un tema pendiente a estudiar en Chile y una problemática con prejuicios y creencias erróneas.

\section{Revolución del alma para quienes mere- cen respeto}

La historia de las personas con discapacidad intelectual ha sido de marginalidad en sus deberes y derechos en el Siglo XXI. A pesar de los esfuerzos, esa situación y creencias de la cultura no han evolucionado. Belmonte y García (2014) plantean que la discriminación es un hecho por tradición o falta de informa- ción, viviendo la vulneración de derechos, discriminación y segregación a nivel social. Mansilla (2006) señala que la persona es un valor en sí misma, a nivel individual y social. La persona es un microcosmos espiritual y no debe ser tratada como objeto de segregación. Por otra parte, Sánchez y Solar (2008) señalan que el modelo social exige enfocar la situación de las personas con discapacidad como un problema de desigualdad y discriminación.

Zárate afirma: "La persona humana, dotada de cualidades y posibilidades, tiene como misión construirse a sí misma y ayudar en la construcción de los otros" (2008, p. 34). Los valores son dinámicos y están ligados al desarrollo de la personalidad, son condicionados por los procesos de socialización, con la presencia de relaciones empáticas hacia el otro.

\section{La dimensión cultural en la afectividad y sexualidad en jóvenes}

Las nuevas formas de relacionarse han generado una revolución de las manifestaciones afectivas. Es evidente que jóvenes con discapacidad intelectual tienen formas de expresarse sin eufemismos, donde los impulsos parecen estar primero debido a que no se le da el espacio adecuado para comunicar y expresar sus sensaciones. Las culturas y creencias de su sistema se vuelven represoras, invasoras y limitantes en la búsqueda del placer.

Amor (2000) plantea que las manifestaciones afectivas desencadenan problemas, más que un atributo favorecedor e inherente de las personas. Se presenta una actitud restrictiva que es justificada por la sociedad con sentimientos de temor, confusión y culpabilidad. Por tanto, es necesario un cambio, considerando una actitud empática, que según Infante (2013) y Stein (1917) cambia la perspectiva del pensamiento, promoviendo el desarrollo del cuerpo, alma y espíritu, permitiendo encontrar la esencia de cada persona. Otro valor es el amor, planteado por Mansilla (2006) como un escenario, que no solo presenta un conglomerado de estados afectivos, se presenta como un fenómeno origina- 
rio, que se puede intuir y nunca definible, orientado a individuos portadores de valores.

Desde el ámbito de la Psicopatología, se describieron alteraciones y trastornos que dan apoyo a la idea de que estados de ánimo positivos y negativos pueden ser experimentados de forma muy intensa (emociones positivas y negativas) en períodos de tiempo muy breves e incluso en el mismo momento ( $\mathrm{Pa}-$ drós, Soriano \& Navarro, 2012).

\section{El cuerpo según la perspectiva de ser en el mundo}

El ser humano, desde el nacimiento hasta la muerte vive en un cuerpo sexuado, que percibe, experimenta, siente y se relaciona consigo mismo y el mundo, construyendo su identidad.

El cuerpo permite adquirir un punto de vista de sí mismo. Por tanto, la afectividad y sexualidad permiten comunicar el cuerpo con el entorno favoreciendo la construcción social de la persona. Caricote (2012) y Vargas Barrantes (2013) señalan que las oportunidades para ampliar el conocimiento con respecto a la sexualidad en personas con discapacidad intelectual se encuentran limitadas, al no considerar roles sociales ni nuevas estrategias de comprensión y significancia en su diario vivir.

Las personas con discapacidad intelectual han pertenecido a las minorías y se les ha negado toda posibilidad de manifestaciones o expresiones intersubjetivas. Por tanto, el objetivo de la investigación realizada fue describir las manifestaciones afectivas de las personas con discapacidad para dejar de lado la privación de estos derechos.

\section{Método}

Esta investigación se orientó desde un paradigma hermenéutico, el que según Guba (citado en Mansilla, 2006) devela la realidad social, construida a través de los significados otorgados por los actores, cuyo proceso es la búsqueda de interpretaciones desde el punto de vista de las personas, con el fin de lograr la comprensión de los fenómenos, revelando la valoración de estudiar la acción y el mundo social desde los significados de las personas implicadas a través de sus creencias, intensiones y motivaciones.

El paradigma hermenéutico permite emitir diferentes técnicas de interpretación de una realidad, es decir, busca descubrir los significados de las distintas expresiones humanas, como palabras, gestos, textos, sin perder su singularidad y en este caso específico, las respuestas y expresiones emitidas por los jóvenes con discapacidad intelectual en sus manifestaciones afectivas.

Esta investigación fue de tipo cualitativo, dado que se buscó interpretar una realidad social. Para Denzin y Lincoln "son prácticas interpretativas que hacen visible al mundo, permiten transformación y representación" (2005, p. 3$)$.

La investigación tiene nivel descriptivo denso. Se considera un estudio descriptivo, debido a que identifica aspectos del fenómeno, para luego detallar y especificar las propiedades significativas que arrojen como resultado del trabajo con los sujetos de estudio. Esta recolección de información se obtiene a través de los relatos de los propios sujetos, en los cuales se intenta comprender el discurso desde el marco de referencia propio de los individuos, experimentando la realidad en el campo de estudio, dando paso a la descripción de lo que se pretende investigar para así caracterizar el fenómeno.

Para el plan de análisis se utilizó el software Atlas.ti versión 8.0, esta herramienta permite expresar el sentido circular del análisis cualitativo y posibilita la incorporación secuencial de datos (San Martín, 2014).

El análisis se efectuó a través de codificación abierta que consiste en dividir y codificar datos en conceptos y categorías. Posteriormente, se realizó la codificación axial y se articularon nuevos datos con las categorías resultantes de anteriores comparaciones. 
Desde la dimensión ética esta investigación se orienta a principios de la declaración de Singapur (2010) promoviendo la integridad con la que se realizó, con honestidad y responsabilidad profesional en relación a los sujetos de estudios y veracidad de los resultados.

\section{Participantes}

Los participantes fueron 13 alumnos de una escuela especial en la Araucanía, con un rango de edad de 15 a 25 años, que presentaban condición de discapacidad intelectual y 4 profesores de educación especial del mismo contexto escolar.

\section{Instrumentos}

Se administró la entrevista semi-estructurada que según Flick (2004) incluye supuestos explícitos e inmediatos, que el entrevistado puede expresar espontáneamente al responder preguntas abiertas.

\section{Resultados}

De la categoría principal Manifestaciones afectivas de los jóvenes con discapacidad intelectual surgieron dos subcategorías o familias de códigos: (1) Relaciones de amistad / Relaciones de pareja y (2) Creencias de los jóvenes / Creencias de profesores.

Subcategoría 1. Relaciones de pareja - Relaciones de amistad (ver Figura 1).

La subcategoría Relaciones de amistad de los jóvenes está asociada con el primer código, Bienestar emocional, provocado por este tipo de relaciones, apareciendo en el siguiente fragmento de la entrevista: "Yo cuando estoy con mis amigos, me siento y me pongo alegre siempre, me da gusto estar con ellos, hacer cosas con ellos" [E8:094].

Paredes (2010) menciona que es una dimensión irreemplazable en la calidad de vida expresada en la afectividad de los jóvenes, para entregar apoyos en el desarrollo personal y las competencias del mismo al interactuar con otros.
El segundo código, Compañía, se evidencia en el siguiente relato: "Estar compartiendo con él y a la vez compartiendo con otros amigos." [E4:061]. Cornejo y Bellon (2001) señalan que la compañía consiste en estar con otro, sentirse protegido y resguardado en situaciones donde necesitan apoyo y comprensión, experimentando lazos afectivos.

El tercer código es Valoración del otro y se evidencia en el siguiente relato:

"Estar compartiendo con él y a la vez compartiendo con otros amigos." [E4:061]. Cornejo y Bellon (2001) señalan que la compañía consiste en estar con otro, sentirse protegido y resguardado en situaciones donde necesitan apoyo y comprensión, experimentando lazos afectivos; "Hablando bien, no andar enojada. No andar mirando a los compañeros enojado, ayudarlo para que no esté triste." [E10:032].

Para Cuervo (2013) se vincula con la consolidación de una comunicación compartida, demostrar preocupación, protección y entrega de afectos en virtud del reconocimiento mutuo, respetando individualidades y espacios compartidos con el amigo, sintiendo apoyo social.

El siguiente y cuarto código corresponde a Posicionamiento valórico que se expresa en: "Portándose bien y no portándose mal." [E6:028]; "preocuparse cada día de hacer buenas acciones, ser bueno" [E9: 049]

Mendoza (2005) menciona que el sistema de valores condiciona las respuestas a patrones sociales éticamente correctos y que en este caso son manifestaciones afectivas acorde a los lineamientos, creencias y discernimiento impuesto por adultos, quienes establecen límites no consensuados con jóvenes y que acomodan a un supuesto bien social.

El quinto código que se desglosa es Materialización del cariño y aparece en el fragmento de entrevista: "Entregándole algún regalo, una rosa, un chocolate." [E1: 117].

Belk y Coon (1993) indican que obsequiar de forma espontánea elementos que representen la valoración por el otro puede significar amor y preocupación por la persona, re- 
presentando significados que se tienen por el ser querido. Además, los jóvenes no solo enuncian su amistad con elementos concretos, sino que involucran Expresiones fisiológicas que se evidencian en: "Si porque entre los dos nos mirábamos, sentía como ansiedad así como que quería decir algo.” [E3:089].

Para Waldstein, Kop, Schmidt, Haufler, Krantz y Fox (2000) es una respuesta autónoma del cuerpo a un evento en particular, que en reiteradas veces son involuntarias e intervienen en el actuar. Se desprende a la vez el séptimo código, Expresiones corporales, que puede leerse en el siguiente fragmento: "Dar caricias, besarse, darse besos, que el novio traiga flores" [E2:059].

Pease, A. y Pease, B. (2012) indican que el lenguaje del cuerpo es un reflejo externo de la condición emocional de la persona; los gestos y movimientos permiten descubrir y expresar emociones.

La subcategoría Relaciones de pareja se asocia al primer código, Valoración personal, que puede leerse en el siguiente fragmento: "Que me haga reír, que me quiera, que me respete y que lo quiero todo, lo quiero con todo mi corazón." [E10:111].

Por tanto, Zárate (2008) señala que la persona está llamada a encontrar su vocación, entonces requiere un conocimiento de sus características que permita comprender su individualidad y autenticidad. Esto se puede vincular a manifestaciones de los jóvenes en sus relaciones de pareja, ya que ellos se comprenden como seres únicos, con cualidades y defectos.

La afectividad se manifiesta a través del segundo código, Expresiones corporales, en el siguiente relato: "Pololear, es acariciarse, cuando otra persona se abraza, se dan besos, salen algún lugar." [E1:058].

Desarrollándose de forma paralela el tercer código, Expresiones fisiológicas, evidenciado en el siguiente relato: "Sí, porque entre los dos nos mirábamos, sentía como ansioso así como que quería decir algo." [E3:089].

Las relaciones afectivas de pareja están ligadas al cuarto código que es Amor y se evidencia en: "Amar y no dejarla nunca, una persona que ama a otra." [E6:181]. Piazzesi
(2015) plantea que es la experiencia personal de sentirse unido, comprometido y conectado a otras personas. Un repertorio personal, pero al mismo tiempo por lo menos parcialmente compartido, de gestos, palabras, acciones u omisiones que expresan el amor o la ausencia de amor. Este repertorio personal se constituye durante el proceso de socialización primaria así como durante las experiencias afectivas, discursivas, culturales de la persona.

De acuerdo a esto, los jóvenes comprenden que la esencia del amor es relacionarse o conectarse con un otro, que proporciona compañía, protección y respeto, bienestar emocional y felicidad.

Las Relaciones de pareja se asocian con el Posicionamiento valórico que lo establecen las creencias que condicionan el discernimiento de los jóvenes al igual que en las Relaciones de amistad y que se presenta en: "Porque no demostraba lo que hacía en la escuela a veces era irrespetuoso y eso a mí no me gusta" [E8:080].

Por otro lado existe un contingente de jóvenes que no han experimentado relaciones de pareja y manifiestan el sexto código que es Incomodidad, señalado en: "Incomoda, no sé, para eso está la casa o no sé, pero es que igual se siente media rara, Si mi mamá dice que no te vea la gente ahí, en la calle es feo dice." [E7:280].

Para Guzmán, Peloquin, Lafontaine, Trabucco y Urzúa (2014) es parte del elemento afectivo, es la tendencia a experimentar incomodidad o malestar al ser testigo de situaciones interpersonales de tensión o pasión.

Además, al buscar pareja muestran el deseo de Idealización de las relaciones por lo que han escuchado o ha sido inculcado por adultos significativos, siendo el séptimo código y que se ve en el fragmento: "Tiene que ser bonito. Si, felices se ríen todo el rato" [E1:260].

Rodríguez, T. y Rodríguez, C. (2002) señalan que se recrea la idealización del ser amado, a partir de historias personales o recreaciones socioculturales de su entorno vin- 
culadas con pasión y desamor, los jóvenes buscan responder al prototipo ideal de pareja y relaciones.

Subcategoría 2. Creencias de Profesores / Creencias de Jóvenes (ver Figura 2)

La subcategoría relaciones de amistad de los jóvenes está asociada con el "Bienestar emocional" provocado por este tipo de relaciones, apareciendo en el siguiente fragmento de entrevista: "Yo no me ponía así, me ponía alegre siempre" [E8:094]. Paredes (2010) menciona que es una dimensión irreemplazable en la calidad de vida expresada en la afectividad de los jóvenes, para entregar apoyos en el desarrollo personal y las competencias del mismo al interactuar con otros. "Que actúe bien, no mal" [E2:360].

El segundo código Conciencia de responsabilidades en el fragmento: "yo tengo claro que primero tengo que terminar mis estudios, tengo que aprender aquí lo que más pueda, ya que eso lo que debo hacer" [E9:394].

Nobleza (2009) plantea que es posible sostener que los nuevos significados atribuidos a la maternidad son una consecuencia del valor social de la misma y el valor afectivo que las madres adjudican a sus hijos o hijas dadas sus historias personales. Sin embargo, la existencia de esta resignificación no implica que las jóvenes se encuentren suficientemente preparadas para acceder a un mundo adulto, sino que la maternidad se convierte en una nueva demanda para las jóvenes madres. Es un proceso que exige compromiso, motivación y recursos económicos, por lo que los jóvenes deben conocer efectos que trae consigo la maternidad.

En relación con la comunicación de los jóvenes y sus manifestaciones afectivas se desprende el tercer código, Ausencia de comunicación familiar, de "No sé, porque con mi familia no converso" [E8:421].

Para Garcés y Palacio (2010, p. 7), los conflictos familiares pueden en determinado momento, convertirse en barreras que limitan la comunicación y las relaciones, cuando se pierde el respeto y el reconocimiento de los demás miembros como legítimos otros en la convivencia se opone a la comunicación "es la tendencia natural de los personas a juzgar, evaluar, aprobar o desaprobar los juicios de otras personas."

Cuando esto ocurre, el conflicto familiar se transforma en una barrera de comunicación, con lo cual se genera una comunicación negativa y limitada, ya que el sujeto intimidado puede perder confianza en sí mismo y en el otro.

Por medio de actitudes de autoritarismo que disminuyen la comunicación familiar y la comprensión entre los integrantes de este núcleo, se genera así desconfianza al no poseer orientaciones del sistema inmediato más significativo, apareciendo el código Subordinación evidenciado en: "No, sería ridículo, tampoco más adelante. Me matan, me voy de la casa antes que me encuentren" [E2:387].

Milgram (1973, p. 6) menciona que "Lo esencial es que la persona llega a considerarse instrumento para realizar los deseos de otra" teniendo una perspectiva diferente donde la persona subordinada siente orgullo o vergüenza al realizar una acción, en este caso los jóvenes obedecen reglas de adultos significativos que condicionan sus manifestaciones afectivas.

La siguiente subcategoría es Creencias de los profesores de acuerdo a las manifestaciones afectivas de los jóvenes, desprendiéndose el primer código, Valoración de la persona, que se ve reflejado en: "Bueno es algo normal, es una situación que tanto personas con o sin situación de discapacidad tienen que vivir" [E2: 33].

Román Ortiz (2012) menciona que el valor de la persona sólo es dado por y en el acto del amor. Se evidencia además el código Confusión concepto amistad reflejado en: "Las relaciones de amistad de los jóvenes no están bien fundamentadas en valores muy estables..." [E2:15].

Lo cual sustentan Rodríguez, T. y Rodríguez, C. (2002) señalando que en ocasiones, los jóvenes suelen definir o valorar la amistad por medio de elementos superficiales, tales como atributos materiales, cercanía o proximidad física, por lo cual, al existir conflictos entre amigos, se genera un quiebre en la relación. 
En tanto el primer código, Compromiso familiar se refleja en lo siguiente: "entonces eso se da aquí entre nosotros, cumplimos muchas veces el rol de papá o mamá, porque también tenemos que educar en lo sexual (...) a quien ellos comunican es a la docente no a los papás." [E2:066].

Para Coutts y Morales (2011, p. 456), "La educación sexual empieza en el hogar." Se logra vincular con el contexto a estos jóvenes, desde la dinámica familiar, quienes omiten la educación en estos temas, llevando así a responsabilizar a las instituciones educativas, con el fin de suplir esta necesidad. De acuerdo a esto, se desprende el segundo código, Infantilización, que queda evidenciado en: "Los padres, todavía visualizan a sus hijos como niños, pese a que son adultos, acá la mayoría tiene sobre 20..." [E2: 066].

Esto refleja la percepción que presentan los padres en relación a sus hijos con discapacidad intelectual, y es avalado por Giami, Glat y Freaitas (1996) citado en Mattos y colaboradores (2012). Los padres idealizan a sus hijos como niños eternos, por presentar una condición diferente, respaldando la falta de preocupación en la afectividad.

El tercer código, Redes de apoyo, se refleja en: "El mismo trabajo interdisciplinario (...) buscar también apoyos en profesionales de otras áreas" [E1:039].

El centro educativo realiza un trabajo con redes de apoyo con las que cuenta, sin embargo, Navarro y Hernández (2012) plantean que no basta con asistir a una institución o especialistas, debe existir un verdadero involucramiento de los padres en la educación sexual de sus hijos.

El cuarto código, Educación sexual transversal, se evidencia en el siguiente texto: "Para escuelas especiales no tienen objetivos claros a la educación sexual, (...) para las características de nuestros estudiantes a veces no está contextualizado" [E3:055].

García, Pérez y Barruezo (2002) plantean que represión y control que instituciones y personas presentan ante las manifestaciones afectivas de las personas con discapacidad intelectual incide en la expresión natural de ser joven.
El quinto código, Aprendizaje experiencial, está expresado en: "En aula siempre se trata desde lo vivencial, en base a la etapa de vida que están pasando los jóvenes" [E3:092].

Según Romero (2010), este aprendizaje ofrece una oportunidad única, ya que se consolida en él un conocimiento significativo, contextualizado a los estudiantes, transferible $\mathrm{y}$ funcional.

El sexto código, Mediación, se evidencia en: "Entonces al verse eso dificultado tenemos que sí o sí que conversarlo con los estudiantes, mediar y ver como se restaura la relación." [E1:009].

Para Ríos Cabrera (2006), en las experiencia debe existir un mediador que apoye y se interponga entre el aprendiz y su contexto, para ayudar a organizar y desarrollar sus aprendizajes.

En tanto el séptimo código, Género, se refleja en la respuesta:

"Claro si me pasa con estudiantes del laboral que vienen a tocar la puerta, mire profesor podemos hablar con usted (...) pero está esta mirada bien machista igual sobre todo con los varones" [E4:097].

Según Stern (2007), la imagen de género es la base del camino, por la cual los adolescentes comienzan a adquirir su identidad, conduciéndoles a interiorizar patrones de comportamiento esperados.

Finalmente, el octavo código, Normas de convivencia, está reflejado en lo siguiente: "el establecimiento posee un protocolo de convivencia escolar para situaciones, complejas y de acuerdo a ello se dan directrices para abordar ciertas temáticas" [E2:057].

Donde Coutts y Morales (2011) señalan que se deben considerar aspectos valóricos y culturales propios de cada persona, una normativa ética y jurídica que limite la conducta y genere la represión de las manifestaciones afectivas.

\section{Conclusiones}

Se logró develar que las manifestaciones de afectividad en los jóvenes con discapacidad intelectual se ven limitadas por las creencias de su contexto inmediato: espe- 
cialmente la familia y la escuela. Son las instituciones que regulan el comportamiento de forma autoritaria, además la presencia excesivamente infantilizada desde los adultos que afecta su espontánea expresión afectiva. La negación de los padres acerca de que sus hijos están en una etapa de juventud $\mathrm{y}$ a que la vivan cualquier joven, les impide comprender que sus hijos sienten la necesidad de manifestarse afectivamente, creando una protección social, ocultando la sexualidad como un proceso biológico y natural, afectando el índice de desarrollo humano de los jóvenes y su autodeterminación.

Los jóvenes asocian las relaciones de amistad con el bienestar personal, compañía, protección, complicidad, respeto y el compartir intereses evidenciando un posicionamiento valórico cultural, que regula y alude al sistema de valores de un ideal de comportamiento.

En sus manifestaciones afectivas predomina el machismo en la asignación de roles, proyección de vida en pareja y formación de familia, develando la conciencia de responsabilidades sociales según lo que se espera éticamente. Además se incluye la maternidad como un deseo a futuro que se ve oprimido por las bajas expectativas de los adultos significativos. La familia se presenta como obstaculizadora en las relaciones afectivas de los jóvenes, evidenciándose así una falta de comunicación y comprensión.

Los profesores consideran a los jóvenes como personas de valor, y que su condición intelectual no afecta sus derechos de manifestarse afectivamente ya sea con sus amigos o parejas. Sin embargo, mencionan la presencia de confusión en el concepto de amistad asociado a la abstracción de esta terminología. Dentro del establecimiento existen normas de convivencia que buscan regular las conductas, establecer límites y reprimir manifestaciones que puedan ser imitadas por niños de otros niveles.
Por esto, es necesario guiar y orientar en este proceso, no solo desde la institución educativa sino en un trabajo conjunto con la familia, donde no deleguen esta formación solamente en los profesionales, que sea un aprendizaje contextualizado de educación sexual y se acepte como una condición inherente del ser humano, que debe experimentar en libertad de derechos.

La educación sexual con respecto a las manifestaciones afectivas de los jóvenes con discapacidad, no solo debe ser abordada biológicamente, debe también considerarse el desarrollo y la formación de actitudes, sentimientos, comportamientos, valores personales, autoimagen y estilo de vida.

La educación sexual debe proporcionar información precisa y no restringirse al compromiso intelectual sino también respetar su edad evolutiva, donde la educación sea precisa y directa, otorgando recursos didácticos que ayuden a que ellos sean conscientes de su actuar. Debe ir acompañada de guía, mediación con respecto a la construcción de vínculos y vivencias afectivas adecuadas, dentro de sus características propias.

Se debe avanzar hacia una educación sexual que promueva el diálogo con los jóvenes, donde se respete lo que piensan y lo que expresan, sus intereses, sus preocupaciones, la responsabilidad de su actuar de manera de manifestarse y asimilar el sentido de respetarse a sí mismo.

A pesar de los avances científicos y el cambio del modelo social de la discapacidad intelectual, aún se vislumbra una sociedad represora orientada a la victimización de la personas por su condición, siendo un tarea pendiente la generación de conciencia social, respeto de derecho y dignificación favoreciendo la autodeterminación en la proyección de vida. 


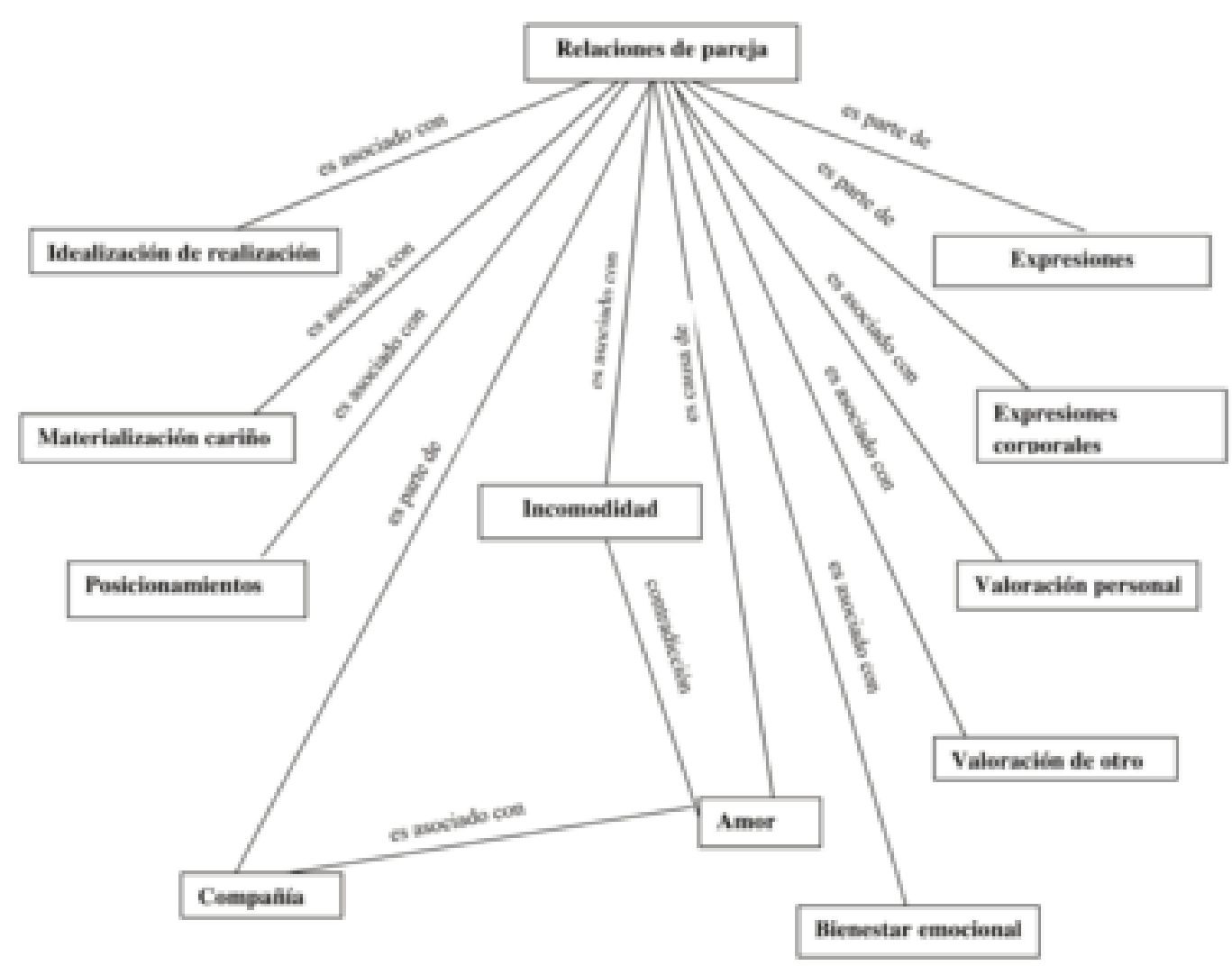

Figura 1. Subcategoría 1. Relaciones de pareja / Relaciones de amistad 


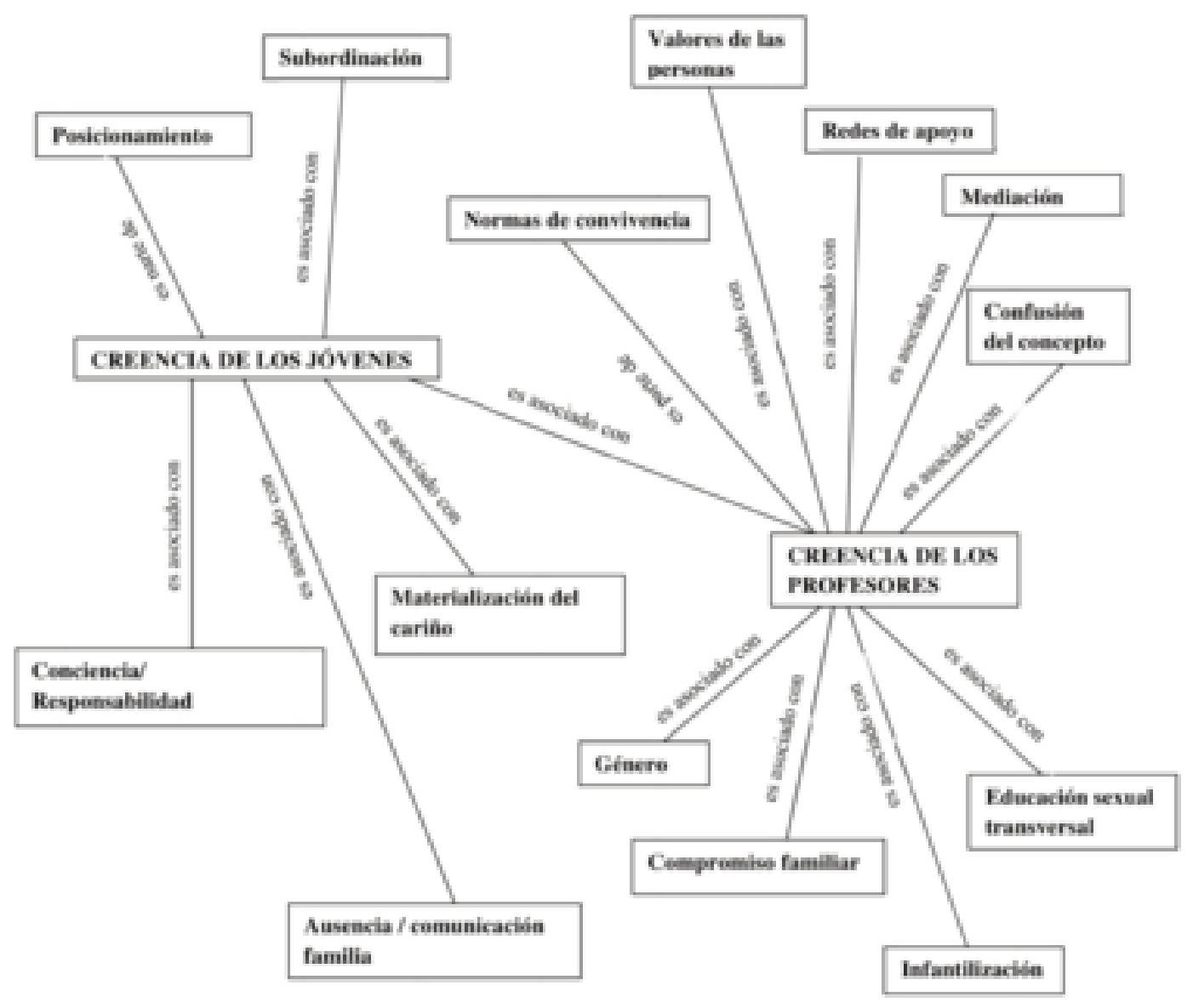

Figura 2. Subcategoría 2. Creencias de Profesores / Creencias de Jóvenes 


\section{Referencias bibliográficas}

Abreu, E. (2010). Socio-cultural paradigm, integration and sexual education in people with Mental Disability. Revista Argentina de Psiquiatría, 90(11), 147-153. Recuperado el 12 de julio de 2016 de http://repositoriocdpd.net: $8080 /$ bitstream/handle/ $123456789 / 786 /$ Art_AbreuGuerra E_ParadigmaSocioCultural_2010.pdf?se quence $=1$

Aguirre, E., Arciniega Buenrostro, L., Galván García, C., Márquez Caraveo, M., Zanabria Salcedo, M. \& Pérez Barrón, V. (2011). Epidemiology and integral domain of intellectual disability. Salud Mental, 34(5), 443-449. Recuperado el 9 de mayo 2016 de http://www.redalyc.org /articulo.oa?id=58221288008

Amor, J. (2000). Sexuality and people with psychic disability. España: FEAPS.

Aya Gómez, L. \& Córdoba, C. (2013). Assuming together the challenges: life qualyty in families of young people with intellectual disability. Revista de la Facultad Médica, 63(2), 80-90. Recuperado el 9 de mayo de 2016 de http://www.scielo.org.co/scielo.php?script=sci arttext\&pid=S0120-00112013000200007

Belk, R. \& Coon, G. (1993). Gift giving as agapic love: An alternative to the exchange paradigm based on dating experiences. Journal of Consumer Research, 20(3), 393-417. Recuperado el 12 de mayo de 2016 de http://www-personal.umich.edu/ prestos/Downloads/DC /Smith_BelkandCo on1993.pdf

Belmonte, M. \& García, M. (2014). Intelectual. The school of life, another look at intellectual disability. Revista de la Facultad de Ciencias de la Educación, 14(1), 147-170. Recuperado el 16 de marzo de $2016 \mathrm{de} \mathrm{http://institucional.us.es/fuentes/ges-}$ tor/apartadosrevista/pdf/campo/cpwalqjn.pdf

Campbell, M. \& Gilmore, L. (2014). The Importance of Social Support for Students with Intellectual Disability: An Intervention to Promote Mental Health and Well-Being. Cypriot Journal of Educational Sciences, 9(1),
21-28. Recuperado el 10 de julio de 2016 de h t t p : / / w w w. w or ld-ed u c at i on center.org/index.php/cjes/article/view/3-3/pdf 249

Caricote, E. (2010). Sexuality in Adolescents with Disabilities. Revista de la Facultad de Ciencias de la Salud, 2(16), 53-57. Recuperado el 25 de septiembre de 2016 de http://www.scielo.org.ve/scielo.php?script=sc i_arttext\&pid=S1316-71382012000200009

Caricote, E. (2012). Sexuality in intellectual disability Educere, 55(16), 395-402. Recuperado el 27 de mayo de 2016 de http://www.redalyc. org/articulo.oa? $\mathrm{id}=35626140020$

Cosentino, S., Maia, K., Rodrigues, M., Rossetto, M. \& Silva, V. (2010). Educational workshops on adolescent sexuality: School as scenario. Revista Electrónica Cuatrimestral de Enfermería, 20(1), 1-8. Recuperado el 7 de julio de 2016 de http://scielo.isciii.es/scielo.php?pid=S1695$1412010000300009 \&$ s cript $=$ sci arttext

Cornejo, I. \& Bellón, E. (2001). Prácticas culturales de apropiación simbólica en el centro comercial Santa Fe. Convergencia. [Cultural practices of symbolic appropriation in the Santa Fe shopping center. Convergence]. Revista de las Ciencias Sociales, 24(8). Recuperado el 27 de septiembre de 2016 de http://www.redalyc.org/articulo.oa? $\mathrm{id}=10502403$

Coutts, W. \& Morales, G. (2011). Sex education should begin at home and continue at school. Revista Chilena de Pediatría, 5(82), 454-460. Recuperado el 2 de junio de 2016 de http://www.scielo.cl/scielo.php?pid=S037041062011000500012\&script $=$ sci_arttext

Cuervo, J. (2013). Viable couples that last in time. Perspectiva Psicológica, 2(9), 257-270. Recuperado el 15 de julio de 2016 de http://revistas.usta.edu.co/index.php/diversitas/article /view/1209/1424.

Chávez, M. \& Álvarez, J. (2012). Psychosocial aspects associated with sexual behavior in young people. Revista Psicología y Salud, 1(22), 8998. Recuperado el 15 de julio de 2016 de http://www.uv.mx/psicysalud/psicysalud-221/22-1/Mayra\%20Ch\%E1vez.pdf 
Denzin, N. \& Lincoln, Y. (2005). The Sage Handbook of Qualitative Research. Londres, Inglaterra: Sage.

Ferreira, W. (2014). Vulnerability to sexual violence in the context of inclusive school: reflection on the invisibility of the disabled person. Revista Latinoamericana de Educación Inclusiva, 2(1), 109-128. Recuperado el 18 de julio de 2016 de http://www.repositoriocdpd.net:8080/bitstream/handle/123456789/113/Art_FerreiraW B_ViolenciaSexualDiscapacidad_2008.pdf?s equence $=1$

Flick, U. (2004). Introducción a la investigación cualitativa. [Introduction to qualitative research]. Madrid: Morata.

García, J., Pérez, J. \& Barruezo, P. (2002). Discapacidad Intelectual: Desarrollo, Comunicación e Intervención. [Intellectual Disability: Development, Communication and Intervention]. Madrid: Cepe.

Garcés, M. \& Palacio, J. (2010). Family communication in subnormal monteria settlements. Psicología desde el Caribe, 25, 1-29.

González, E., Molina, T., Montero, A. \& Martínez, V. (2011). Factors associated with sexual initiation in adolescents of both sexes of middle-low socioeconomic level of the Metropolitan Region. Revista Chilena de Obstetricia y Ginecología, 78(1), 4-13. Recuperado el 20 de julio de 2016 de http://www.scielo.cl/pdf/rchog/ v78n1/art02.pdf

Guzmán, M., Peloquin, K., Lafontaine, M., Trabucco, C. \& Urzúa, A. (2014). Evaluation of the dyadic empathy: Analysis of the psychometric properties of the Interpersonal Reactivity Index in Couples (IRIC-C) in the Chilean context. Psicoperspectivas, 13(2), 156-164. https://doi.org/10.5027/psicoperspectivasvol13-issue2-fulltext-340

Infante, F. (2013). Ficción en la idea de empatía de Edith Stein. [Fiction in the idea of empathy by Edith Stein]. Ideas y valores, 67(53), 137-155. Recuperado el 2 de agosto de 2016 de
http://www.eleutheia.ufm.edu/Articulos 140120 CRLo pez_Stein.htm

Janeiro, J., Oliveira, I., Rodrigues, M., Maceiras, M. \& Rocha, G. (2013). Sexual and contraceptives attitudes, the locus of health control and self-esteem among higher education students. Revista Bras Promoc Saude Fortaleza, 26(4), 503-509.

Katz, G. \& Lazcano, E. (2008). Sexuality in subjects with intellectual disability: an educational intervention proposal for parents and counselors in developing countries. Salud Pública de México, 50(2), 239-254. Recuperado el 15 julio de 2016 de http://www.scielosp.org/pdf/spm/v50s2/a $18 \mathrm{v} 50 \mathrm{~s} 2 . \mathrm{pdf}$

Kijak, R. (2011). A Desire for Love: Considerations on Sexuality and Sexual Education of People With Intellectual Disability in Poland. Sex Disabil, 1(29), 65-74. Recuperado el 24 de julio de 2016 de http://www.ncbi.nlm.nih.gov/pmc/articles/PMC3034884/pdf/11195_2010_Article 9184. pdf

León, D. (2012) Afectividad y conciencia: la experiencia subjetiva de los valores biológicos. [Affectivity and consciousness: the subjective experience of biological values]. Revista Chilena de Neuropsicología, 7(3), 108-114. Recuperado el 29 de Octubre de 2016 de http://www.redalyc.org/articulo.oa?id=1793249 86003

León, L., Bolaños, G., Campos, J. \& Mejías, F. (2013). Percepción de una muestra de educandos y docentes sobre la implementación del programa de educación para la afectividad y sexualidad integral [Perception of a sample of learners and teachers on the implementation of the education program for afectivity and integral sexuality]. Revista Electrónica Educare, 17(2), 145-165. Recuperado el 14 de junio de 2016 de http://www.redalyc.org/articulo.oa?id $=194127506010$

Malón, A. (2012). The right to a sex education? Between the discourses of salvation and the 
absence of knowledge? Revista Educación siglo XXl, 2(30), 207-228. Recuperado el 6 de marzo de 2016 de http://revistas.um.es/educatio/article/view/1537 61/ 140801

Mansilla, J. (2006). El corazón bien ordenado. [The well-ordered heart]. Salamanca: UPSA. Mattos Caldeira, P., Rajab Cardia, D., Bicalho Reis, L. \& Da Silva Ferrao, E. (2012). Sexualidade na deficiência intelectual: uma análise das percepções de mães de adolescentes especiais. [Sexuality in intellectual disability: an analysis of the perceptions of special adolescent mothers]. Revista Brasilera de Educación Especial, 3(18), 469-486. Recuperado el 24 mayo de 2016 de http:// www.scielo.br/scielo.php?pid=S1413$65382012000300008 \& \mathrm{script}=\mathrm{sci}$ arttext\&tlng=pt

Mateos Inchaurrondo, A., Balsells Bailón, A., Molina Garúz, M., Fuentes Peláez, N., Pastor Vicente, C. \&Amorós Martí, P. (2014). Educational needs to promote affective and sexual health in atrisk youth. Revista d'Innovació $i$ Recerca en Educació, 7(2), 14-27. Recuperado el 15 de julio de 2016 de http:/www.raco.cat/index.php/REIRE/article/view/278670/366416

Mella, J. (2012). Essay on the importance of sex education in people with intellectual deficit: from occupational therapy. Revista Chilena de Terapia Ocupacional Facultad de Medicina Escuela de Terapia Ocupacional, 12(2), 1-13. Recuperado de http://www.anales-ii.ing.uchi le.cl/index.ph p/R T O/article/ viewFile/25311/26633

Mendoza, J. (2005). El profesor y la formación valórica. Reflexiones y aportes. Perspectiva Educacional, 45(2), 61-71. Recuperado de http://www.redalyc.org/pdf/3333/3 33328 803005.pdf

Milgram, S. (1973). Obedience to authority. An experimental point of view. Bilbao, España: Desclee de Brouwer S.A.

Mora Huerta, C. \& Rodríguez Calvillo, M.A. (2011). Sexuality, disability and an unexpected population. An issue less controversial every day. Revista electrónica de Psicología, 14(3), 27-36. Recuperado el 24 de julio de 2016 de https:/www.revistas.unam.mx/ index.php/repi/article/download/27645/25597

Navarro, Z. \& Hernández, I. (2012). Influence of parents' attitudes towards sex education and intellectual disability. Revista Psicología y Salud., 22(2), 195-203. Recuperado el 2 de octubre de 2016 de http://www.uv.mx/psicy$\mathrm{s}$ a $1 \mathrm{ud} / \mathrm{p}$ s i c y s a $1 \mathrm{ud}-22-2 / 22-$ $2 / \mathrm{Nub}$ i a $\% 20 \mathrm{Zu} 1$ e i m a $\% 20$ Navarro\%20Guadarrama.pdf

Olavarrieta Bernardino, S., Besteiro López, B., Darin, L., Suárez López, P., Tur Bonnin, N. \& Gómez-Jarabo Gómez, G. (2013). Attitudes towards sexuality, sterilization, maternity / parenting and parenting skills of people with intellectual disabilities: a preliminary study. Siglo Cero: Revista Española sobre Discapacidad Intelectual., 44(4), 55-69. Recuperado el 9 de septiembre de 2016 de http://www.researchgate.net/publication/259618207

Padrós, F., Soriano, C. \& Navarro, G. (2012). Positive and negative affect: one bipolar dimension or two independent unipolar dimensions? Interdisciplinaria, 29(1), 151-164. Recuperado el 13 de de septiembre de 2016 de http://www.scielo.org.ar/pdf/interd/v29n1/ v 29 n 1 a 09 .pdf.http:// dx.doi .org/ 10.1016/j.beth.2017.07.003

Pallisera Díaz, M., Fullana Noell, J., Martin Pazos, R. \& Vilá Suñé, M. (2013). Transition to the adult life of young people with intellectual disabilities. The opinion of professionals in school and post-school services. Revista Española de Orientación y Psicopedagogía, 24(2), 100-115. Recuperado el 11 de octubre de 2016 de http://www.redalyc.org/articulo.oa?id=338230794007

Panksepp, J. (2011). The basic emotional circuits of mammalian brains: ¿Do animals have affective lives? Neuroscience and Biobehavioral Reviews., 35(9), 1791-1804. Recuperado el 22 de julio de 2016 de http://www.researchgate.net/profile/Jaak_Panksepp/ publication/516 00666 
Paredes, D. (2010). Bienestar emocional y expresión conductual en las personas con discapacidad intelectual [Emotional well-being and behavioral expression in people with intellectual disabilities]. Revista Educación $y$ Diversidad, 4(2), 51-60. Recuperado el 30 de septiembre de 2016 de https://dialnet.unirioja.es/servlet/articulo? codigo $=3276331$

Pava-Ripoll, N. (2015). Conversational narratives with families and teachers of children with disabilities: a methodological contribution. Interdiscipinaria, 32(2), 203-222. Recuperado el 17 de octubre de 2016 de http://www.redalyc.org/articulo.oa?id=18043 528001

Pease, A. \& Pease, B. (2012). Conecta: Los secretos del lenguaje corporal del amor. Barcelona: Amat editorial.

Piazzesi, Ch. (2015). Producir una mirada sociológica sobre el amor [Produce a sociological look about love]. Revista Latinoamericana de Estudios sobre Cuerpos, Emociones y Sociedad, 7(18), 8-18.

Reisin, S. (2010). Vivienda autónoma: un dispositivo para la inclusión social de jóvenes y adultos con discapacidad intelectual [Autonomous housing: a device for the social inclusion of young people and adults with intellectual disabilities]. Revista Vertex de Psiquiatría, 21(90), 141-146. Recuperado el 12 de diciembre de 2016 de http://bibliomedicinadigital.fmed.uba.ar/med i c i n a / c g i b i n/library.cgi ? $\mathrm{a}=\mathrm{d} \& \mathrm{c}=$ catalogo $\& \mathrm{~d}=$ Catalogo Revar_ 33741

Ríos Cabrera, P. (2006). La aventura de conocernos. [The adventure of knowing each other]. Caracas: Textos.

Rodríguez, T. \& Rodríguez, C.(2002) .T. El amor y las nuevas tecnologías experiencias de comunicación y conflicto. Revista Comunicación y Sociedad. 1(25), 15-51. Recuperado 29 de septiembre de 2016 de http://www.redalyc.org/articulo.oa?id=34642628002

Roman, Ortíz, A. D. (2012). Value and education of love according to Max Scheler and St.
Augustine of Hipponna. C.E.E.O., 1, 74-89. Recuperado en 24 de julio de 2016 de http:// cejsh.icm.edu.pl/cejsh/element/bwmeta1.../Arti cle-Roman-Ortiz.pdf

Romero, M. (2010). Experiential learning and new training demands. Revista de Antropología Experimental., 10(8), 89-102. Recuperado el 18 de junio del 2016 de http://revista.ujaen.es /huesped/rae/articulos2010/edu1008pdf.pdf

San Martín, D. (2014). Theoretical theory and Atlas.ti: methodological resources for educational research. Revista Electrónica de Investigación Educativa., 16(1), 104-122. Recuperado el 9 de Julio de 2016 de http://redie.uabc.mx/vol16no1/contenido-sanmartin.html

Sánchez,. O. y Solar. I. (2008). The International Convention on the Rights of Persons with Disabilities and its impact on the autonomous legislation of Cantabria. España: DYKINSON.

Silveira, Araújo, G, M. y Rossito, Aiello, A, L. (2013). Rede Social de Apoio de Mães com Defi ciência Intelectual. Rev. Psicologia: Refl exão e Crítica., 26 (4) 752-761. Recuperado el 15 de julio de 2016 de http://www. scielo.br/pdf/prc/v26n4/16.pdf

Sim-Sim, M. (2013). Pasión y experiencias sexuales en jóvenes portugueses. Revista de Psicología para América Latina. 24, 64-82. Recuperado 29 de septiembre http://www.revistapsicolatina.org/wp-content/uploads/2013/08/Revista_24.pdf

Stein, E. (1917). Sobre el problema de la empatía. [On the problem of empathy]. Madrid: Trotta.

Stern, C. (2007). Gender stereotypes, sexual relations and adolescent pregnancy in the lives of young people from different sociocultural contexts in Mexico. Estudios Sociológicos., 73 (25) 105-129. Recuperado en http://dialnet.unirioja.es/servlet /articulo? codigo $=2381743$

Tabernero, M. \& Politis, D. (2016). Recognition of basic emotion and their relation with theory of mind in the behavioral variant of frontotemporal dementia. Interdisciplinaria, 33(1), 21-39. 
Recuperado el 27 de julio de 2016 de http://www.scielo.org.ar/pdf/interd/v33n1/v33n 1a02.pdf

Uribe, J, I., Amador, G., Zacarías, X. y Villarreal, L. (2012). Perceptions about condom use and sexuality among young people. Revista Latinoamericana de Ciencias Sociales, Niñez y Juventud., 10 (1) 481-494. Recuperado en 27de julio del 2016 http://www.scielo.org.co/scielo. php? pid=S 1692-715X2012000100031 \&script $=$ sci_arttext

Vargas Barrantes, E. (2013). Sexuality as a human expression evaluated in young people and teachers from a public school in the canton of Alajuela Costa Rica. InterSedes., 15 (30) 22-54. Recuperado en 13 de Octubre del 2016 http://revistas.ucr.ac.cr/index.php/ intersedes/art icle/view/14868/14145

Vianchá, A., Bahamón, J, Tobos, R., Alarcón,. L. y Uribe, Alvarado, J. (2012). Sexuality in young people: An analysis from the model ecological. Psychological theses. Tesis psicológicas, 7(2), 74-89. Recuperado el 28 de septiembre de 2016 de http://publicaciones.libertadores. edu.co/index.php/Tesis Psicologica /article/view/274
Viera Cherro, M. (2011). Let them know. Body and sexuality in social zoom Revista Estudios Feministas., 19(2) 351-369. Recuperado en 22 de Octubre del 2016 http://www.scielo. br/pdf/ref/v19n2/ v19n2a03.pdf

Vived, Conte, E., Mullet, E., Díaz, Orgaz, M., González, Sanz, A. y Matía, Amor, A. (2013). Advancing towards independent living: educational approaches in young people with intellectual disabilities Revista Española de Discapacidad., 1 (1) 119-138. Recuperado en 27 de septiembre en $2016 \mathrm{http}: / /$ dialnet.unirioja.es/servlet/articulo?codigo $=4868169$

Waldstein, S., Kop, J., Schmidt, A., Haufler, J., Krantz, S. \& Fox, A. (2000). Frontal electrocortical and cardiovascular reactivity during happiness and anger. Biological Psychology, 55(1), 3-23. Recuperado el 28 de julio de 2016 de http://citeseerx.ist.psu.edu /viewdoc/download?doi=10.1.1.319.6217\&rep $=$ rep1\&type $=$ pdf

Zárate, I. (2008). Report on special educational needs. Guanajuato, México: Edissa.

Fecha de recepción: 6 de julio de 2016 Fecha de aceptación: 28 de diciembre de 2017 
